\title{
Colloidal dispersions of octadecyl grafted silica spheres in toluene: A global analysis of small angle neutron scattering contrast variation and concentration dependence measurements
}

\author{
Joachim Kohlbrecher \\ SINQ, Paul-Scherrer Institut, 5232 Villigen PSI, Switzerland \\ Johan Buitenhuis, Gerhard Meier, ${ }^{\text {a) }}$ and M. Paul Lettinga \\ IFF, Weiche Materie, Forschungszentrum Jülich, Postfach 1913, 52425 Jülich, Germany
}

(Received 26 April 2006; accepted 13 June 2006; published online 28 July 2006)

\begin{abstract}
In this paper we report measurements of the form factor and the structure factor of a sterically stabilized colloidal dispersion consisting of silica spheres coated with octadecane in toluene by small angle neutron scattering (SANS). The phase diagram of this system shows the liquid-liquid coexistence line and also a jamming transition at higher concentrations, where the jamming line intersects the coexistence line roughly at the critical point. We have performed SANS experiments at a temperature well above the transition temperature and at various volume fractions $\phi$, spanning from the very dilute regime $(\phi=0.2 \%)$ to the critical concentration $(\phi=16 \%)$ and the highly viscous regime $(\phi=39.2 \%)$. Except for the very dilute regime, we observe a structure factor $S(q)$ in all other cases. We fitted our data over the whole concentration regime using a global fitting routine with a core-shell model for the form factor $P(q)$, taking into account the structure factor, which we describe with the Robertus model for an adhesive polydisperse core-shell particle. At a volume fraction of $\phi=5 \%$ a SANS contrast variation experiment has been performed. From that the product of the volume of the shell and the amount of solvent within the corona of our core-shell particle could be determined. At the most probable shell thickness of $2.3 \mathrm{~nm}$ a solvent content of about 50\% within the corona was found. Moreover we could conclude that the core is not interpenetrated by solvent molecules. From the contrast variation experiment followed that the structure factor at zero average contrast exhibits a strong $q$ dependence, which is an effect of an inhomogeneous particle in combination with a size distribution. (C) 2006 American Institute of Physics. [DOI: 10.1063/1.2220564]
\end{abstract}

\section{INTRODUCTION}

A very successful theory to describe colloidal systems is the so-called hard sphere theory, in which particles interact only at the closest approach via an infinitely steep pair potential. Here the particle radius is simply set by excluded volume only. ${ }^{1}$ In cases where the potential rises not that steep, like a Yukawa-type potential, ${ }^{2}$ the effective hard sphere diameter can be adjusted or equivalently the effective volume fraction. This still allows for a reliable estimation of the equilibrium structure and thermodynamic properties. The situation is getting more complex, if one adds a short range attractive interaction or potential to the hard sphere model. This potential is called a square well potential or specifically the Baxter model for an adhesive hard sphere $^{3}$ and is sketched in Fig. 1 schematically. Systems, which can be described with such a potential, show rich phase behavior, if the attractive part is strong enough. Examples for those systems are sterically stabilized colloids in marginal solvents, which were reported for example, by Jansen et al. ${ }^{4}$ Edwards et al., ${ }^{5}$ and Rouw and de Kruif. ${ }^{6}$ The model system we use for our investigations was introduced by de Kruif and

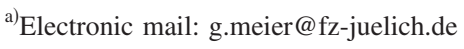

Schouten $^{7}$ and consists of silica spheres grafted with octadecyl chains in toluene. However, also the use of other marginal solvents has been reported. ${ }^{8-10}$

In toluene at a volume fraction of about $\phi=0.1$ a liquidliquid phase transition is observed, in a very convenient temperature and pressure range. In the case of sterically stabilized colloids the nature of the interaction in the hairy corona of the silica spheres will change with pressure and temperature $^{7}$ and a liquid-liquid phase transition will take place. The interaction between spheres is short ranged; therefore we can assume that its relevant length scale is at most as long as the corona thickness, which is in the order of $2 \mathrm{~nm}$, as concluded from geometric considerations. Most probably the structure of the corona, and to a lesser extent the silica core, plays a key role in the liquid-liquid phase transition. ${ }^{4}$ Knowledge of these features can be obtained from small angle neutron scattering (SANS) as has been shown in numerous other investigations on core-shell particles, ${ }^{11-15}$ since it can provide us with the desired information by performing contrast variation experiments. However, an unambiguous interpretation of such data is not straightforward. Therefore it is the main objective of this paper to present a most detailed investigation of the equilibrium configuration of our system. The SANS data will yield structural information on length 


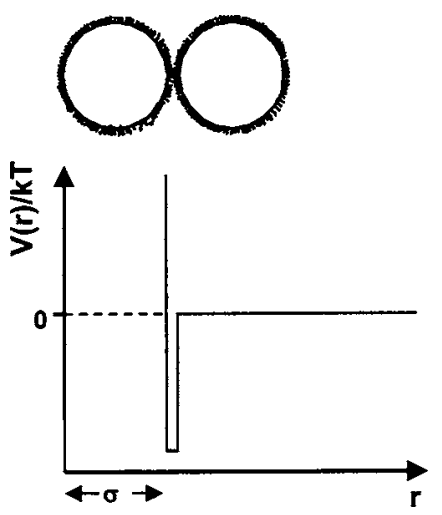

FIG. 1. Pair interaction $V(r)$ between two adhesive hard spheres with diameter $\sigma$ and a square well with width $\Delta$ and depth $\varepsilon$.

scales comparable to the size of the spheres used in our experiments, being about $80 \mathrm{~nm}$ in diameter and its corona thickness. Thereby we take advantage of the fact that the scattering length density of the silica core can be matched by a proper mixture of protonated and deuterated solvent such that only the corona is visible and hence subtle changes there are well detected. Two important features come up while dealing with properties of the corona of a core-shell particle: its thickness and its possible penetration by solvent molecules. In addition to that the grafting density is important, since both parameters characterize the effective scattering length density, which is essential to know in performing a numerical analysis of the scattering data in order to extract the thickness. In this paper we will first characterize our system, i.e., the phase diagram as obtained by "simple" visual inspection, the SANS experiments to determine the form and structure factors and a contrast variation to explore the solution properties in more detail with the aim to learn something about the layer thickness and its penetration by solvent molecules. To this end we will employ a global fit routine, which allows us to use fit parameters simultaneously for a whole series of data curves and is thus capable to provide information which would not be possible to obtain from a single curve fit alone. Last but not least we have investigated the volume fraction dependence of the intensity to measure the structure factor and will analyze the data on the basis of various models for $S(q)$ including the Baxter model, thereby using an algorithm for the numerical calculation of the structure factor for a polydisperse system. ${ }^{16}$ The paper is organized as follows: In Sec. II we will present the experimental methods used, synthesis, transmission electron microcopy (TEM), light scattering (LS), and SANS. In Sec. III A we will present the phase diagram leading over to the SANS: Basic formulas are presented in Sec. III B, followed by results, first the structure factor, Sec. III C, and then the contrast variation, Sec. III D. We conclude with Sec. IV.

\section{EXPERIMENT}

\section{A. Synthesis and elemental analysis}

Silica core particles were prepared according to Stöber et $a .^{17}$ and then grafted with stearyl alcohol following van Helden et al. ${ }^{18}$ to obtain octadecyl chains anchored onto the surface. The dispersion was purified by repeated cycles of centrifugation and redispersion in chloroform and cyclohexane. Stock solutions were prepared by drying the dispersion under a nitrogen flow at about $50{ }^{\circ} \mathrm{C}$ and immediately followed by redispersing in toluene-h8 and toluene-d8. All further samples were prepared by pipetting from these two stock solutions. The concentration of these solutions was determined by drying a small volume of dispersion to constant weight at $150{ }^{\circ} \mathrm{C}$. After storage for some longer time the concentration of the stock solutions was checked.

The volume fraction of the dispersions was obtained from the weight concentration and the density of the particles. The density of the particles was determined from the density of a dispersion with a concentration of $0.665 \mathrm{~g} / \mathrm{ml}$. This was done by weighting $10 \mathrm{ml}$ of the dispersion as well as the solvent toluene-h8 in the same measuring flask. Assuming additivity of volumes of solvent and particles, which is a good assumption for these colloidal dispersions, a density of the particles of $\rho_{p}=1.78 \mathrm{~g} / \mathrm{ml}$ was obtained.

Elemental analysis was performed by the Central Division of Analytical Chemistry ( $\mathrm{ZCH})$ of the Forschungszentrum Jülich on a LECO CHNS-932 analyzer. The sample was dried for $24 \mathrm{~h}$ at $50{ }^{\circ} \mathrm{C}$ under vacuum. An average carbon content of $9.5 w / w \%$ was obtained, which is attributed to $11.1 \mathrm{w} / \mathrm{w} \%$ alkyl chains. Assuming additivity of volumes and a density of $0.78 \mathrm{~g} / \mathrm{ml}$ for the alkyl chains and $2.0 \mathrm{~g} / \mathrm{ml}$ for the silica a volume fraction of $24.3 \%$ of alkyl chains in the particles can be estimated from this weight concentration. This value will be compared to the SANS result later.

\section{B. Sample characterization by TEM and LS}

We have performed static (SLS) and dynamic (DLS) light scattering as well as TEM on our samples. The light scattering was performed on a custom-made goniometer, which allows us to measure the static and dynamic light scattering in an angular range of $\theta=30^{\circ}-120^{\circ}$. The concentration of spheres used for the light scattering experiments was $1 \mathrm{~g} / 1$. As a result from dynamic light scattering experiments, a single exponential decay of the intensity autocorrelation function and a decay rate proportional to square of the scattering vector are obtained. From that a diffusion coefficient of $D=9.84 \times 10^{-12} \mathrm{~m}^{2} / \mathrm{s}$ is calculated which in turn gives a radius of $r=39.6 \mathrm{~nm}$ via the Stokes-Einstein relation. Likewise the radius of the particles can be obtained from a Guinier analysis of the total scattered intensity $I(q)$ as a function of angle. From the slope of the Guinier plot a radius of $(5 / 3)^{1 / 2} r_{G}$ was calculated, which gives $r=43 \mathrm{~nm}$ in fair agreement with the result from the dynamic measurement.

TEM has been performed on a Philips CM200 microscope at a voltage of $200 \mathrm{kV}$. A drop of $1 \mathrm{~g} / 1$ solution was put on a regular TEM grid and was transmitted after force drying without further masking. In Fig. 2 the histogram of the size distribution obtained from our TEM data is shown. All together 177 particles have been analyzed by standard image processing software, resulting in a mean diameter of $\langle d\rangle=67 \mathrm{~nm}$ with a relative standard deviation of $\sigma=0.13$. On comparing the average diameter from TEM with the values from LS, one has to consider that TEM is sensitive on the 


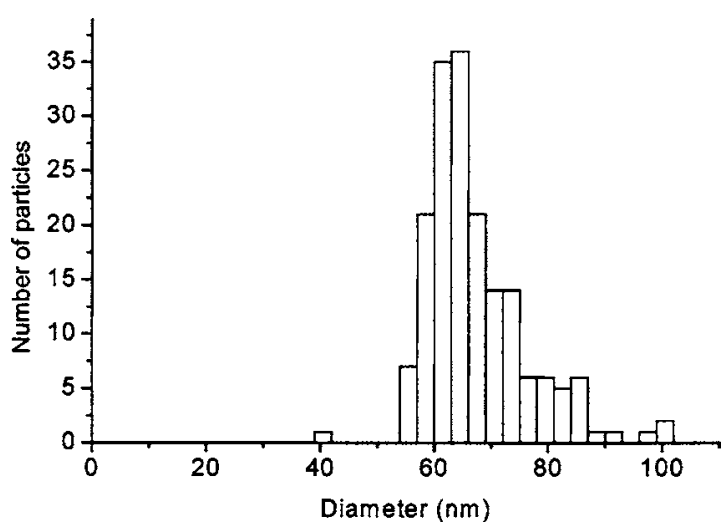

FIG. 2. Size distribution as obtained from a TEM analysis of 177 silica particles.

number average diameter of the particles, whereas dynamic light scattering is sensitive to a higher order average; specifically, it measures the sixth moment of the inverse $z$-averaged diameter. Inspection of Fig. 2 shows that the distribution is asymmetric towards higher diameters. We find different values for $d$ obtained from light scattering and from TEM. For the here obtained standard deviation of $\sigma=0.13$ the ratio in diameters according to Thomas ${ }^{19}$ should be $d_{\mathrm{LS}} / d_{\mathrm{TEM}}=1.22$. We find $83 / 67=1.24$, which is in good agreement. A source of uncertainty stems from the fact that the contrast in TEM from the corona of the coated silica particles is poor, since it contains only carbon and hydrogen atoms, which scatter much less than silicon, being located in the core. Therefore the obtained TEM diameter is at maximum reduced by doubling the corona thickness, which is about $4 \mathrm{~nm}$. Taking that into account we reach about $71 \mathrm{~nm}$ for the diameter, which gives then a ratio of the different moments of $83 / 71=1.17$, also in fair agreement with the expectation.

\section{SANS experiment}

We have performed the SANS experiments at the SANS I machine at the SINQ spallation source at the PSI in Villigen, Switzerland. ${ }^{20}$ We used thermal neutrons of wavelength $\lambda=0.8 \mathrm{~nm}$ having a wavelength spread $\Delta \lambda / \lambda$ of about 0.1 . The data analysis was performed using the BERSANS software package, ${ }^{21}$ which accounts for all necessary corrections due to background, transmission, count rate/dead time ratio, masking, and radial averaging of the raw data. A standard water sample was used for calibration to absolute scattering intensities and also to account for a nonuniform detector efficiency. ${ }^{22}$ All scattering curves were fitted with theoretical expressions thereby taking the resolution of the spectrometer into account using a procedure described by Pedersen et al. ${ }^{23}$ We have measured at two different detector positions, 18 and $6 \mathrm{~m}$, which give access to a $q$ range of $0.02<q / \mathrm{nm}^{-1}<1.2$, where $q$ is the scattering vector, defined as $q=|\mathbf{q}|=(4 \pi / \lambda) \sin (\theta / 2)$, with $\lambda$ being the neutron wavelength and $\theta$ being the scattering angle.

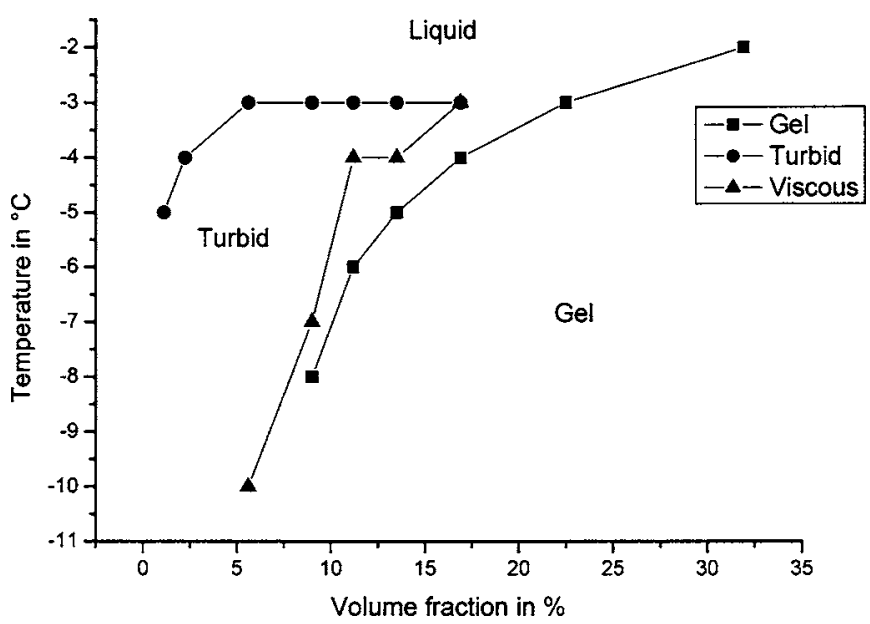

FIG. 3. Phase diagram of silica spheres coated with octadecane in toluene. The lines are guide to the eye.

\section{RESULTS AND DISCUSSION}

\section{A. Phase diagram}

Our system, octadecyl grafted silica in toluene, was chosen in analogy to octadecane coated silica in benzene, which was described by Verduin and Dhont ${ }^{24}$ and de Kruif et al. ${ }^{8,9}$ The main difference between these systems is a shift of the coexistence line with respect to temperature. de Kruif and Schouten ${ }^{7}$ have found for the above mentioned silica system in toluene a $T_{\text {coex }}$ of about $6{ }^{\circ} \mathrm{C}$, whereas we find that our toluene system phase separates at a lower temperature $\left(T_{\text {coex }}=-3{ }^{\circ} \mathrm{C}\right.$ at $\left.\phi=0.13\right)$. We found this $T_{\text {coex }}$ by visual observation of the phase behavior of our colloidal system changing temperature and concentration, as shown in Fig. 3. The difference is probably due to different grafting densities or roughness of the particles on the length scale of the coating layer thickness. In Fig. 3 three different lines are shown: one is the turbid line, which is a signature of the coexistence line (binodal). Besides the latter thermodynamic information, two other, kinetic items are depicted: First, the viscous line, obtained from the experimental observation, which shows that upon cooling the system towards a temperature which is below that line leads to a drastic increase of viscosity, but the system still flows, if the tube is tilted; and second, the gel line, obtained from the observation, which shows that upon crossing this particular line the system does not flow anymore.

We have performed scattering experiments at various volume fractions starting from $0.2 \%$ for the determination of the form factor, then $5 \%, 11.2 \%, 16 \%$, and $39.2 \%$. For all these latter volume fractions we observe a structure factor. However, it becomes most pronounced at the highest volume fractions, at $39.2 \%$. A quantitative analysis in terms of a sticky hard sphere structure factor for polydisperse systems has been carried out for different models for the structure factor, which is shown later in the paper. The temperature at which we performed our SANS experiments was about $16{ }^{\circ} \mathrm{C}$. From our phase diagram, Fig. 3, it is obvious that our scattering data at that latter temperature are taken fully in the homogeneous phase. This may be completely different for data at higher pressures. De Kruif and Schouten ${ }^{7}$ have re- 
ported the pressure dependence of the transition temperature at $\phi=0.13$ to be $d P / d T_{\text {trans }}=77 \mathrm{bars} / \mathrm{K}$. We have found the same value for our silica/toluene system, ${ }^{25}$ and thus the vicinity of the turbid line by applying pressures of $1-2$ kbars at $16^{\circ} \mathrm{C}$ can easily be reached. We find further that at a volume fraction of about $15 \%-20 \%$, according to Fig. 3 , the gel line seems to intersect the coexistence line. This finding is in agreement with the phase diagram of the related silica/ benzene system obtained by Verduin et al. ${ }^{10}$ and also in agreement with simulations by Miller and Frenkel. ${ }^{26}$ The fact that the gel line intersects the coexistence line is of great practical importance, since it offers the possibility to study the phase transition on a long time scale, probably much longer than it would take without the action of a gelled (percolated or jammed) system.

\section{B. SANS: The form factor for a core-shell particle}

We consider the case of a dilute suspension of noninteracting colloidal particles in the absence of multiple scattering and incoherent scattering. "Noninteracting" specifically means that there is no phase coherence between different particles. For details of the theory see the general references of Higgins and Benoit ${ }^{27}$ and/or Feigin and Svergun. ${ }^{28}$ The total macroscopic cross section $d \Sigma / d \Omega(q)$, measured in a SANS experiment, is given by

$$
\frac{d \Sigma}{d \Omega}(\mathbf{q})=N_{p} F(\mathbf{q})^{2}
$$

where $N_{p}$ denotes the number density of particles and $F(\mathbf{q})$ the single particle amplitude or form amplitude which is defined as

$$
F(\mathbf{q})=\int\left[\rho(\mathbf{r})-\rho_{m}\right] \exp (i \mathbf{q r}) d \mathbf{r} .
$$

$\rho_{m}$ is the scattering length density of the medium. The form factor $P(q)$ for a spherical object like here is simply given by $P(q)=|F(\mathbf{q})|^{2}$ and furthermore $q=|\mathbf{q}|$. In the paper by Pedersen $^{29}$ many expressions for $P(q)$ for different particle shapes are given. In the case of a sphere with a homogeneous scattering length density the expression for the form amplitude reduces to

$$
F(q, r)=V\left(\rho_{c}-\rho_{m}\right) \frac{3[\sin (q r)-q r \cos (q r)]}{(q r)^{3}} .
$$

We deal with core-shell particles, which consist of an inner core with radius $r_{c}$ and uniform scattering length density $\rho_{c}$. The core is surrounded by a shell of uniform thickness $\Delta r$ and scattering length density $\rho_{s}$. For this model the scattering length density profile is given by

$$
\rho(r)= \begin{cases}\rho_{C} & \text { for } r \leqslant r_{C} \\ \rho_{S} & \text { for } r_{C}<r<r_{C}+\Delta r\end{cases}
$$

Solving Eq. (2) for the above scattering density profile yields an expression for the particle form amplitude $F_{\mathrm{cs}}(q)$ of a core-shell particle:
TABLE I. Values of the coherent scattering length densities $\rho$ in $10^{10} \mathrm{~cm}^{-2}$ for all involved compounds. As can be seen, it is possible to contrast match the silica core by a proper mixture of deuterated and protonated toluene. The scattering length density of the corona is negative and cannot be matched by any combination of the two toluenes. The value for silica depends on its density and water and ethanol contents and is thus not exactly known.

\begin{tabular}{lc}
\hline Compound & $\rho$ in $10^{10} \mathrm{~cm}^{-2}$ \\
\hline toluene-d8 & 5.66 \\
Silica & $\approx 2.4$ (found experimently) \\
& $(2.7$ theoretical value) \\
toluene-h8 & 0.94 \\
Octadecane & -0.23 \\
\hline \hline
\end{tabular}

$$
F_{\mathrm{cs}}(q)=\left(\rho_{c}-\rho_{s}\right) F\left(q, r_{c}\right)+\left(\rho_{s}-\rho_{m}\right) F\left(q, r_{c}+\Delta r\right),
$$

where $r=r_{c}+\Delta r$ is the total radius of core plus shell and the $F(q, r)$ is given by Eq. (3). The philosophy of Eq. (5) is illustrated by the gedanken experiment, that in order to calculate $F_{\text {cs }}(q)$, first the form amplitude of a sphere with $\rho_{s}$ and radius $r$ is calculated. This is the second term in Eq. (5). From that sphere we have to subtract the sphere with radius $r_{c}$ and $\rho_{s}$ (the core) and replace it by a sphere with the same radius but a proper $\rho_{c}$. This is the first term in Eq. (5). In a contrast variation experiment, where the scattering length density of the solvent $\rho_{m}$ is varied, different situations can be obtained: If we chose $\rho_{s} \approx \rho_{m}$ (which means, we match out the shell), then the second term in Eq. (5) is zero and we observe the form amplitude of the core. On the other hand, if $\rho_{c} \approx \rho_{m}$ (we match out the core), then only distribution which describes the form amplitude of the shell remains. This regime is therefore most sensitive for obtaining information on the scattering length distribution within the shell structure. Our colloidal particle system under study is dissolved in toluene, which might be either protonated or deuterated. In Table I the respective scattering length densities of the constituents are listed. One can see that there is, according to Table I, no combination of solvents which will mask out the shell. But since the core can be matched out, the shell alone can be nicely seen. The other extreme is that in full $d$-toluene contrast mainly the overall outer dimensions are seen. Since our particles have a size distribution we assumed a SchulzZimm distribution for the overall radius $r=r_{c}+\Delta r$, whereas the corona thickness is supposed to be fixed. This distribution function $L(r)$, which we have to insert in Eq. (5) for $r_{c}+\Delta r$, reads

$$
L(r)=\frac{N}{R_{a}}\left(\frac{r}{R_{a}}\right)^{k-1} k^{k} \exp \left(-k \frac{r}{R_{a}}\right) \frac{1}{\Gamma(k)} \quad \text { with } k=\frac{1}{\sigma^{2}},
$$

where the normalization is given by $\int_{0}^{\infty} L(r) d r=N$. The variance $\sigma$ is a measure of the width of the distribution, $R_{a}$ is a scaling parameter, which defines the maximum of the size distribution for small values of $\sigma$, and $\Gamma$ denotes the gamma function. Then the final expression for the determination of the form factor is given by Eq. (7), which is equivalent to the coherent macroscopic cross section $d \Sigma / d \Omega(q)_{\text {coh }}$ plus an incoherent macroscopic cross section $d \Sigma / d \Omega_{\text {inc }}$, which does 
not depend on $q$. Thus the measured intensity $I(q)$ is given by

$$
I(q)=\frac{d \Sigma_{\mathrm{total}}}{d \Omega}(q)=\frac{d \Sigma_{\mathrm{coh}}}{d \Omega}(q)+\frac{d \Sigma_{\mathrm{inc}}}{d \Omega}=N_{p}\left\langle F_{\mathrm{cs}}^{2}(q)\right\rangle+I_{\mathrm{inc}},
$$

where $N_{p}$ denotes the number density of particles, $I_{\text {inc }}$ is the $q$-independent background, and the $\langle\cdots\rangle$ denotes the average over the particle sizes. Equation (7) is furthermore not valid at higher volume fractions. Then the assumption of no interactions between spheres leading to Eq. (1) is no longer valid. At higher concentrations we observe interference between different particles which thus leads to the development of a structure factor $S(q)$ (see Sec. III C).

\section{SANS: Systems with structure factor $S(q)$}

If the assumption that there is no phase coherence between different particles leading to Eq. (1) is not valid, then we have to extend Eq. (1) for these contributions stemming from different particles $i, j$ :

$$
\frac{d \Sigma}{d \Omega}(\mathbf{q})=\frac{1}{N_{p}} \sum_{i, j}^{N_{p}} F_{i}(q) F_{j}^{*}(q) \times \exp \left[i \mathbf{q r}_{i j}\right]
$$

Here the $F$ 's are the scattering amplitudes of particles $i$ and $j$.

$$
\frac{d \Sigma}{d \Omega}(\mathbf{q})=\sum_{i}^{N_{p}} F_{i}^{2}(q)+\frac{1}{N_{p}} \sum_{i \neq j}^{N_{p}} F_{i}(q) F_{j}^{*}(q) \times \exp [i \mathbf{q r}],
$$

where the first term is the scattering intensity of noninteracting particles (limit of dilute system). The second term describes the interference effects between different particles. In case that the particle size is independent of the particle position we can separate the averaging over the form factors from the averaging over the positions (decoupling approach),

$$
\begin{aligned}
\frac{d \Sigma}{d \Omega}(q) & =N_{p}\left\langle F^{2}(q)\right\rangle+\frac{1}{N_{p}}\langle F(q)\rangle^{2} \sum_{i \neq j}^{N_{p}} \exp \left[i \mathbf{q} \mathbf{r}_{i j}\right] \\
& =N_{p}\left\langle F^{2}(q)\right\rangle+N_{p}\langle F(q)\rangle^{2}[S(q)-1],
\end{aligned}
$$

where $S(q)$ is the structure factor for radial symmetric interactions. It depends only on the arrangement or positions of the particles. That in turn reflects the interaction potential between particles. For some interaction potentials such as sticky hard spheres $S(q)$ can be obtained analytically. For a dilute system the structure factor is identical to 1 and hence Eq. (7) follows from Eq. (10) (except $I_{\text {inc }}$ ). There is a fundamental problem involved in the experimental determination of $S(q)$. Strictly speaking $S(q)$ can only be determined if the two averages $\left\langle F^{2}(q)\right\rangle$ and $\langle F(q)\rangle^{2}$ are the same, which is normally not the case. An exception would be a solution of identical spherical symmetric particles. However, in our case we are always faced with a size distribution and therefore the two averages are not identical. Thus to obtain the effective structure factor $\widetilde{S}(q)$, the concentrated system is measured and is divided by the dilute case and normalized with the concentration,

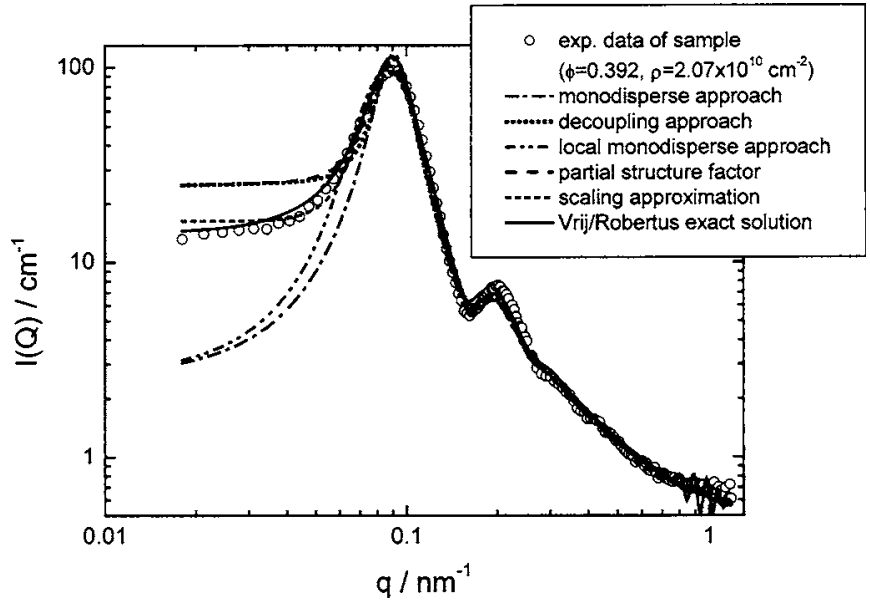

FIG. 4. Comparison of experimental data (black squares) obtained by SANS from a colloid solution with volume fraction $\phi=0.392$ and at a scattering length density of the solvent of $\rho=2.07 \times 10^{10} \mathrm{~cm}^{-2}$ with different models for the structure factor (as listed in the inset) using a polydisperse core-shell form factor, as given by Eq. (5). Only the scaling approximation and the exact solution describe the data well.

$$
\widetilde{S}(q)=\frac{I(q)_{\text {conc }}}{I(q)_{\text {dilute }}}=1+\frac{\langle F(q)\rangle^{2}}{\left\langle F^{2}(q)\right\rangle}[S(q)-1] \stackrel{\text { mono.,ident. }}{\longrightarrow} S(q) .
$$

The above described decoupling approach assumes, that the interaction potential and therefore also the structure factor $S(q)$ are the same for all pairs of particles, independent of their size. However, this is only a sufficient good approximation, when the size distribution is very narrow. Several approaches have been suggested (see Ref. 29 and references therein) to take also different interaction potentials between particles of different sizes into account. A rigorous treatment of the problem is described by $\mathrm{Vrij}^{30}$ for polydisperse hard spheres, which has been extended to polydisperse sticky hard spheres by Robertus et al. ${ }^{16}$ A numerically more simple to implement approach (scaling approximation) has been suggested by Giacometti and co-workers. ${ }^{31,32}$ We have tested all three approaches to our data set. It turned out that for below $5 \%$ all three approximations yield sufficiently good results. For higher concentrations it is, however, necessary to use either the rigorous approach from Vrij or the one from Gazzillo et al., which gives almost the same result. In Fig. 4 we have compared all approaches with each other and with our experimental data obtained at the highest concentration and at a scattering length density of the solvent of $\rho=2.07$ $\times 10^{10} \mathrm{~cm}^{-2}$. We find that except for the scaling approximation and the exact solution, all other approximations show deficiencies either in the intensity at low $q$ and/or value for the first maximum and the width of the peak. The data shown in Fig. 4 were taken at a contrast slightly off the match point, since we had to adjust the scattered intensity to avoid multiple scattering. We are, however, interested in the behavior of our system at the match point, since here we expect the system to be most sensitive on changes in the corona. In order to find out how the structure factor of the system at high concentrations depends on the scattering length densities of the solvent around the zero average contrast condi- 


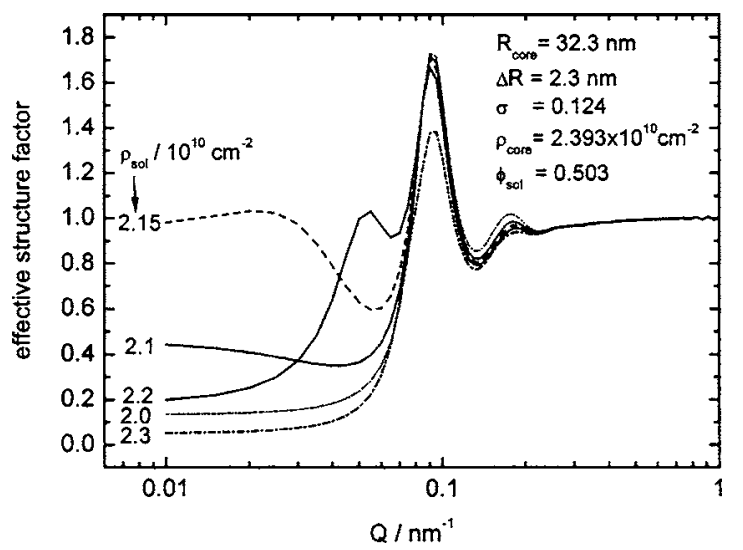

FIG. 5. Calculation of $\widetilde{S}(q)$ for parameters as given in the figure at a volume fraction $\phi=0.392$ (upper right inset, from global fit, see Fig. 7) using the Robertus model ${ }^{16}$ for the structure factor calculated for different scattering length densities of the solvent as indicated on the left. The large increase of $\widetilde{S}(q)$ at low $q$ is most pronounced at zero contrast conditions.

tion, we have calculated the structure factor on the basis of the Robertus model ${ }^{16}$ for different values of $\rho_{\text {sol. }}$. This is shown in Fig. 5, where the parameters used are given in the figure. Clearly we observe a strong increase of intensity for low $q$ around the match point (about $2.15 \times 10^{10} \mathrm{~cm}^{-2}$ ), which means that $\widetilde{S}(q)$ depends strongly on the zero contrast condition. Obviously we observe shape fluctuations, since at zero contrast we are very sensitive on small deviations from the match point condition. This $q$ dependence is thus an effect of an inhomogeneous particle in combination with a size distribution. It also means that it is difficult to analyze data around zero average contrast in terms of $I(q \rightarrow 0)$ and stickiness, because that also would lead to an increase of intensity for low $q$. Therefore, determining the effective structure factor close to the match point is very sensitive to small errors in the scattering length density. As the analysis on the level of form factors and structure factors separately seems to be artificial anyway, we will in the next section present the results of the contrast variation on the basis of the total intensity $I(q)$ and not on the structure and form factor separately. We take structure factor effects into account using the Robertus approach ${ }^{16}$ and the form factor from the model for the core-shell particle [Eq. (5)].

\section{SANS: The contrast variation}

Our particles are core-shell particles, where the respective scattering densities $\rho$ of the core, shell, and surrounding solvent are given in Table I. We have performed most of our experiments by measuring $I(q)$ of our silica particles at a concentration of 5\% in a mixture of $h$ - and $d$-toluene around zero average contrast, in order to find a compromise between sufficient intensity on the one hand and not too large influence of a structure factor on the other hand. According to Table I, there is no combination of solvents, which will mask out the shell. But since the core can be matched out, the shell alone can be nicely seen. A contrast variation experiment has been carried out to get a more detailed picture of the inner structure of the colloid, especially about the shell, i.e., about its thickness and also about the amount of solvent in it. The

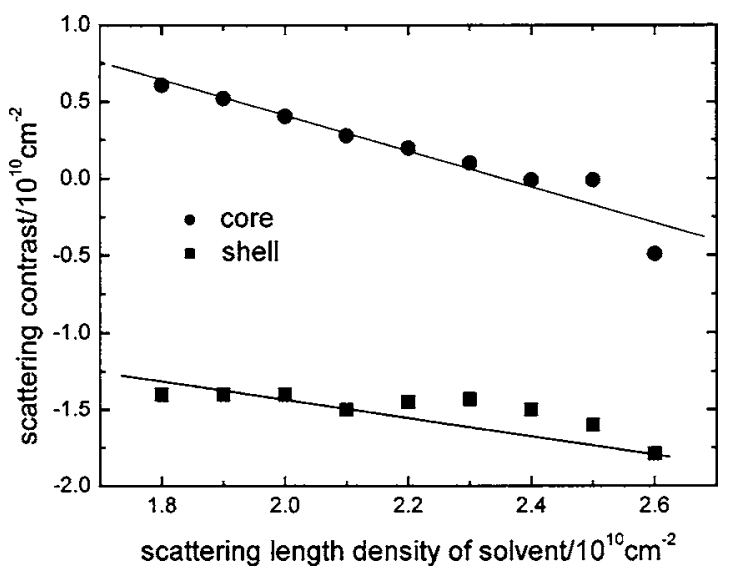

FIG. 6. Plot of the scattering contrasts (circles for core and squares for corona) obtained from single curve fitting of the $5 \%$ data with Eq. (7) with a fixed size distribution $\sigma=0.124$ and fixed shell thickness of $\Delta r=2 \mathrm{~nm}$ vs scattering length densities of the solvent. $S(q)$ was taken into account on the basis of the decoupling approach [Eq. (10)] using $r_{c}=33.6 \mathrm{~nm}, \rho_{c}=2.7$ $\times 10^{10} \mathrm{~cm}^{-2}$, and $\rho_{s}=-0.234 \times 10^{10} \mathrm{~cm}^{-2}$.

contrast variation has been performed at scattering length densities of the solvent ranging from $1.8 \times 10^{10}$ to 2.5 $\times 10^{10} \mathrm{~cm}^{-2}$ in incremental steps of $0.1 \times 10^{10} \mathrm{~cm}^{-2}$. In a first attempt each curve was fitted with Eq. (7) in combination with Eq. (10), where we have fixed the width of the size distribution to $\sigma=0.124$ and the shell thickness to $\Delta r=2 \mathrm{~nm}$. The results for the so obtained scattering length densities for the core and shell are shown in Fig. 6.

From Fig. 6 several important conclusions can be drawn: First of all the so obtained core scattering length density as a function of the scattering contrast is a linear function of the scattering length density of the mixed solvent we used. Moreover the slope is -1 , which indicates strongly that the core is not interpenetrated by solvent molecules. Further evidence comes from the scattering length density of the solvent for which the scattering contrast of the core is zero. This gives a value for $\rho_{\text {silica }}$ of $2.4 \times 10^{10} \mathrm{~cm}^{-2}$ which can be compared to calculations from its chemical composition. The nominal density of silica is $\rho_{p}=2.2 \mathrm{~g} / \mathrm{cm}^{3}$. It is known, however, that Stöber silica particles contain water and ethanol, which in turn decreases the particle density $\rho_{p}$. Here values ranging from $\rho_{p}=(1.8 \pm 0.1) \mathrm{g} / \mathrm{cm}^{3}$ to $\rho_{p}=(2.0 \pm 0.05) \mathrm{g} / \mathrm{cm}^{3}$ have been reported, ${ }^{33,34}$ in agreement with our density value. Using therefore the reasonable assumption that our Stöber particles are well described by a chemical composition such as $\left(\mathrm{SiO}_{2}\right)_{6} \mathrm{H}_{2} \mathrm{O},{ }^{35}$ with a mean density of $\rho_{p}=1.9 \mathrm{~g} / \mathrm{cm}^{3}$, yields a scattering length density of $\rho=2.81 \times 10^{10} \mathrm{~cm}^{-2}$. An addition of $3 \%$ ethoxy groups, which is thought to be reasonable, ${ }^{35}$ decreases the scattering length to $\rho=2.7 \times 10^{10} \mathrm{~cm}^{-2}$. Still from our fit (see Fig. 6) we get a value of $\rho_{\text {silica }}=2.4 \times 10^{10} \mathrm{~cm}^{-2}$. The so obtained value is significantly smaller (about 11\%) than the calculated one for the (scattering length) density of silica. On the other hand the slope of -1 confirms that the core is not solvent interpenetrated.

The results for the scattering length density for the shell scatter more, mainly due to the fact that the shell is rather thin as compared to the diameter of the particle and hence its characterization is more difficult. Moreover a rather unphysi- 

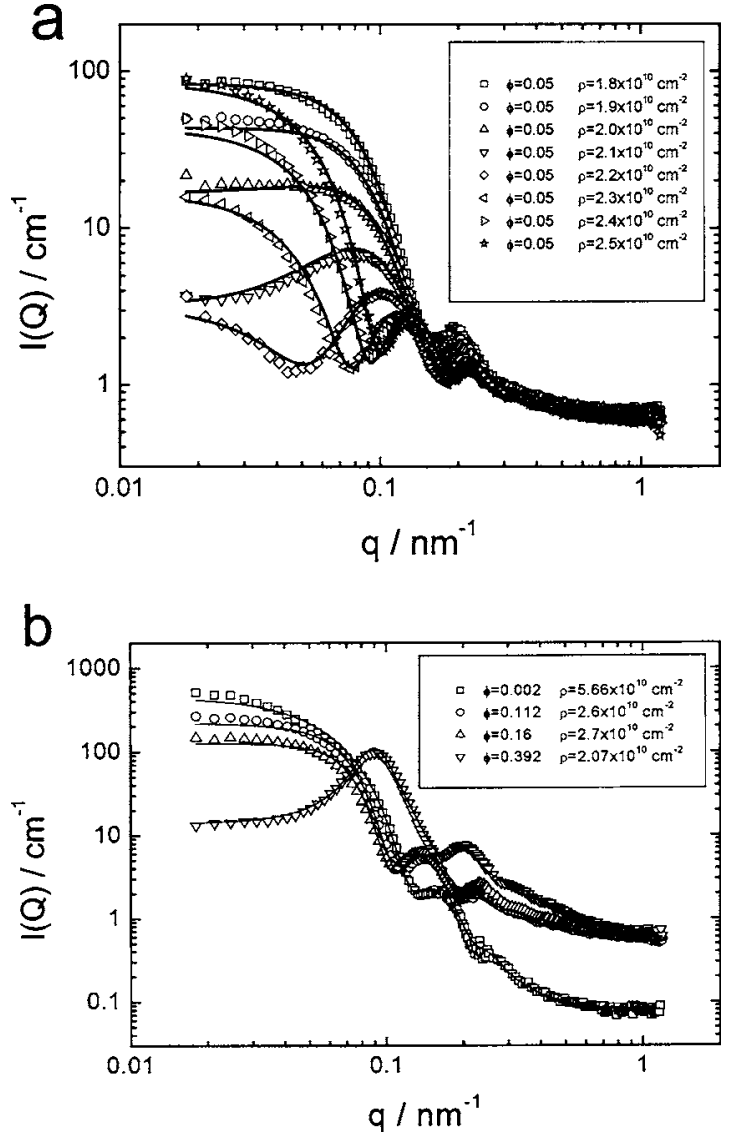

FIG. 7. (a) Fit of Eqs. (6) and (7) and structure factor according to Eq. (10) to the experimental data points obtained by SANS from a 5\% solution at various contrasts around the match point as indicated in the upper right inset. Around $\rho=(2.1-2.2) \times 10^{10} \mathrm{~cm}^{-2}$ the match point is obtained (lowest intensity for vanishing $q$ ) and at $\rho=2.4 \times 10^{-10} \mathrm{~cm}^{-2}$ the core is matched which highlights the form factor of a hollow sphere. (b) Fit of Eqs. (6) and (7) to the experimental data points obtained by SANS from a $0.2 \%$ solution of coated silica spheres in $d$-toluene (top curve, which reaches for high $q$ the lowest intensity, because it is measured in pure $d$-toluene) and fit of Eqs. (6) and (7) and structure factor according to Robertus et al. (Ref. 16) to three higher volume fractions, where the development of the structure factor can be observed. In the inset the volume fractions and scattering length densities of the solvent are listed. The latter three curves reach for high $q$ a kind of plateau, which stems from the incoherent scattering from a part of the solvent, $h$-toluene.

cal independence of the scattering contrast on the scattering length of the solvent was found. This becomes even more evident if we add the value for the contrast of the pure octadecane layer $\left(\rho_{\text {oct }}=-0.234 \times 10^{10} \mathrm{~cm}^{-2}\right)$ to the plot at zero solvent, which acts as a constraint in performing a linear fit to the shell contrast fit values. Then the fit yields a reasonable linear dependence with a slope of -0.61 , which indicates penetration of the layer by solvent molecules. The degree of penetration can be estimated by assuming a linear dependence of the shell scattering length density with $\phi_{\mathrm{sol}}$, the volume fraction of solvent in the shell. Accordingly we write

$$
\rho_{\mathrm{s}}=\phi_{\mathrm{sol}} \rho_{m}+\left(1-\phi_{\mathrm{sol}}\right) \rho_{\text {oct }} .
$$

For this particular case, assuming the shell thickness to be $2 \mathrm{~nm}$, a mean $\phi_{\text {sol }}$ of 0.4 is obtained.

In summary we conclude, that the single curve fitting yields rather difficult to interpret results, since it needs to fix
TABLE II. Parameters of global fit.

\begin{tabular}{lllll}
\hline \hline$r_{c}(\mathrm{~nm})$ & $\sigma_{c}$ & $\Delta_{r}(\mathrm{~nm})$ & $\rho_{c}\left(10^{10} \mathrm{~cm}^{-2}\right)$ & $\phi_{\mathrm{sol}}$ \\
\hline 32.3 & 0.124 & 2.3 & 2.393 & 0.503 \\
\hline \hline
\end{tabular}

parameters in order to get the fits to converge. We have carefully checked whether keeping various parameters fixed (solvent penetration and $\Delta r$ not obtainable separately with confidence) will change the results such that a more physical picture emerges, but that was not the case. Therefore we decided to develop a program in order to be able to handle all the different constrains and also to get a coherent result for the description of the scattering intensities. The program can fit simultaneously all data sets with a global set of parameters. This allows us to separate the influence of several parameters on the intensity signal, which would otherwise be strongly correlated parameters. In the so-called global fitting routine, we used as free parameters in the fit only $r_{c}, \sigma_{c}, \Delta r$, $\rho_{c}$, and $\phi_{\text {sol }}$ according to Eqs. (7) and (12). The scattering length density of octadecane $\rho_{\text {oct }}$ was kept fixed for each global run to $\rho_{\text {oct }}=-0.234 \times 10^{10} \mathrm{~cm}^{-2}$. The data at $5 \%$ in volume fraction were fitted with regard to $S(q)$ assuming a decoupling approach using a fixed hard sphere radius of $r=35 \mathrm{~nm}$. Figures 7(a) and 7(b) show the results of the global fit and the parameters are listed in Table II.

At this point we refer back to Fig. 5, where we had calculated the Robertus structure factor ${ }^{16}$ for different values of the solvent scattering length density around zero contrast. There we found a strong increase of $S(q)$ at zero contrast, being about $\rho_{m}=2.15 \times 10^{10} \mathrm{~cm}^{-2}$. From the inspection of our experimental data in Fig. 7(a) we find the match point at about $(2.1-2.2) \times 10^{10} \mathrm{~cm}^{-2}$, in agreement with that former finding. The intensity $I(q)$ decreases for small $q$ at zero contrast, although $S(q)$ increases (see Fig. 5). The reason is that the form factor for a core-shell particle at zero contrast decreases stronger for small $q$, so that the intensity drops. We had seen that the contrast variation in combination with a single curve fitting was not able to allow a separate determination of the amount of solvent in the shell and the shell thickness (see Fig. 6). Now we carried out the global fitting for several values of the shell thickness. It has been found that as long as the product of $\left(1-\phi_{\mathrm{sol}}\right)$ times the volume of the shell $V_{\text {shell }}$ was kept constant, the resulting scattering curve did not change significantly. Hence $V_{\text {shell }}\left(1-\phi_{\text {sol }}\right)$ $=$ const or equivalently $\Delta r\left(1-\phi_{\text {sol }}\right)=$ const (for $\Delta r \ll r_{c}$ ) gives a hyperbolic relation between $\Delta r$ and $\phi_{\text {sol }}$. To prove this, we performed a fit for several fixed values of the shell thicknesses and plotted the amount of solvent $\phi_{\text {sol }}$ as a function of the shell thickness. The result is shown in Fig. 8 together with the $\chi^{2}$ value of the fit. The plot shows that practically without a loss in the fit quality we always could adjust the amount of solvent, when we changed the value of the shell thickness in the fit such that $V_{\text {shell }}\left(1-\phi_{\text {sol }}\right)=$ const. Furthermore the global fit supports the results from the single curve fitting (see Fig. 8), as it shows that for a $\Delta r$ of $2 \mathrm{~nm}$ indeed $\phi_{\text {sol }}$ is about 0.4 (see Fig. 8). However, without global fit this result would have been thought to be obtained by chance. Moreover, the important relation between volume and 


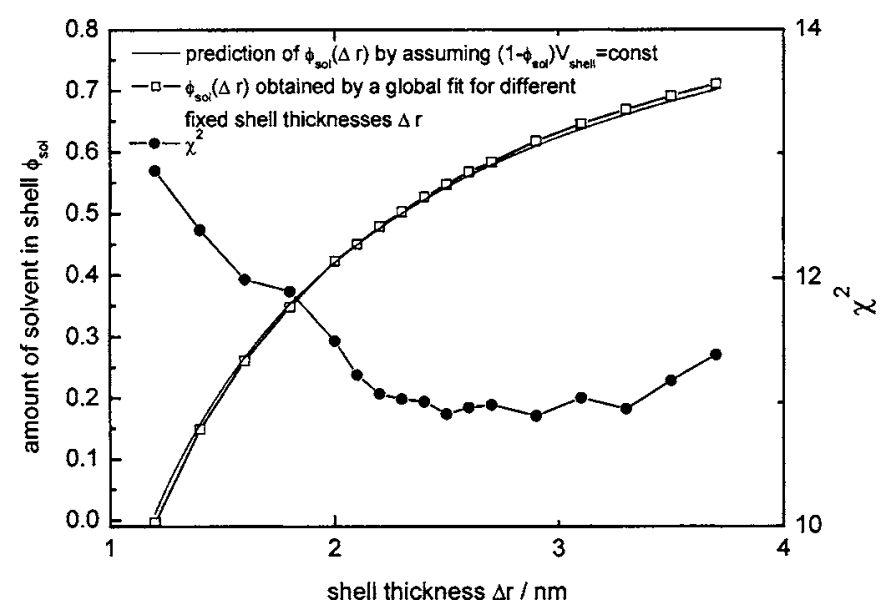

FIG. 8. Correlation between shell thickness and amount of solvent in the shell. The product of $V_{\text {shell }}\left(1-\phi_{\text {sol }}\right)=$ const and thus a hyperbolic dependence between $\Delta r$ and $\phi_{\text {sol }}$ is expected. The open symbols are results from global fit, whereas the full line is expected hyperbolic dependence. Also shown as full symbols is the quality of the fit. The best fit is obtained for shell thicknesses around $2.3 \mathrm{~nm}$.

$\left(1-\phi_{\text {sol }}\right)$ would not have been established. To distinguish between the influences of these two parameters, one needs to measure the coherent scattering intensity at large scattering vectors $q$ to determine $\Delta r$, which is not possible because the measured intensity for large $q$ is dominated by the incoherent background of the sample. It can be shown that based on bond length calculations a $\mathrm{C}_{18}$ alkyl chain has in the stretched configuration a length of about $2.3 \mathrm{~nm}$. This value corresponds roughly to the minimum of $\chi^{2}$ as shown in Fig. 8 and corresponds thus to a solvent penetration of $50 \%$ not too far from the value of $40 \%$ as obtained from single curve fitting.

Another important information, which can be obtained from Fig. 8, is given by the value of the shell volume when there is no solvent in it. That value is proportional to the shell thickness under conditions of no penetration and amounts to $1.25 \mathrm{~nm}$. Given the core diameter distribution, the total amount of octadecyl chains in the shell of the particles as obtained from SANS is $10.3 \%$ by volume. By elemental analysis on dried particles, however, a total volume fraction of alkyl chains of $24.3 \%$ was determined. This finding is supported by comparing the Raman region around $3000 \mathrm{~cm}^{-1}$ of a concentrated silica suspension in toluene with a dilution series of octadecane in toluene from about 70 down to $5 \mathrm{wt} \%$. From an integration of the band intensities in that particular wave number regime we could estimate the amount of methyl(ene) groups to be in the order of $8-10$ wt $\%$, which corresponds to a similar volume of alkyl chains as from elemental analysis. Because it is not possible to obtain a good global fit of the SANS data with a much different amount of octadecyl chains in the shell, the only explanation seems to be that the remaining amount of alkyl chains of $14 \%$ by volume is located in the core of the particles. This can be checked by calculating $\rho_{c}$ for this composition and comparing it to the value obtained from the fit of the SANS data. If we calculate $\rho_{c}$ for a core composed of $15.6 \%$ alkyl chains $(15.6 \%$ alkyl chains in the core corresponds to $14 \%$ of the complete particle) and $84.4 \%$ of silica

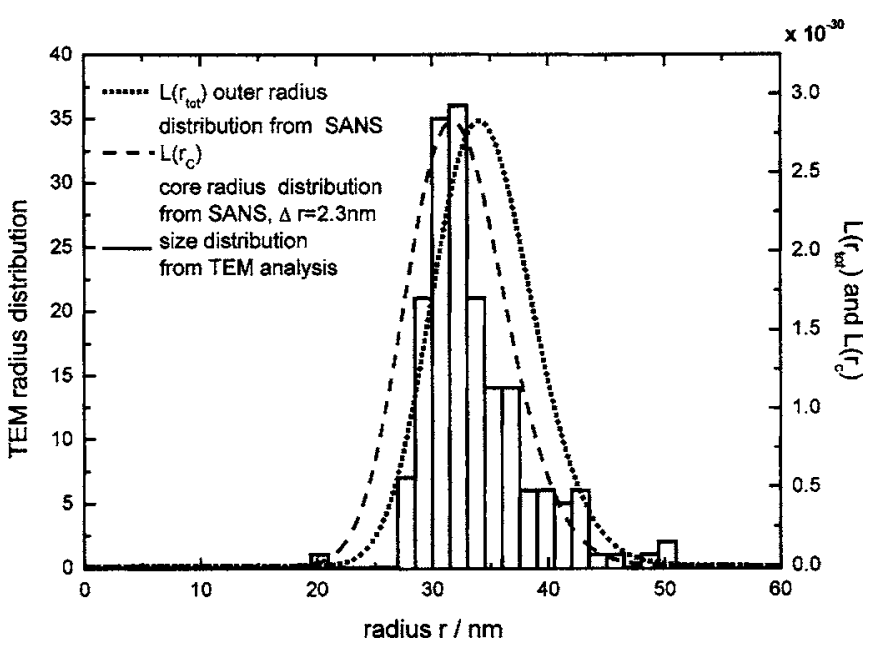

FIG. 9. The size distribution as obtained from the global fit of the SANS results compared to the size distribution as obtained by TEM.

with a composition of $6\left(\mathrm{SiO}_{2}\right) \mathrm{H}_{2} \mathrm{O}$ (Ref. 35) and a density of $2.0 \mathrm{~g} / \mathrm{ml}$, we obtain $\rho_{c}=2.45 \times 10^{10} \mathrm{~cm}^{-2}$. Indeed, this is in excellent agreement with the $\rho_{c}$ of $2.41 \times 10^{10} \mathrm{~cm}^{-2}$ as obtained from the SANS analysis, giving a strong indication that some organic material is contained throughout the silica core. These alkyl chains in the core are thought to be ethoxy groups from the Stöber synthesis or the start of the grafting procedure, or octadecyl chains entering the core during the grafting at $190-200{ }^{\circ} \mathrm{C}$. These results on the grafting density also agree with the recent estimate of the grafting density of $\sim 1 / 3$ octadecyl chains and $2 / 3$ solvent for octadecyl grafted silica particles in hexadecane as studied with the nonlinear optical technique vibrational sum frequency scattering. ${ }^{36}$ As elemental analysis of this type of colloidal particles often results in grafting densities of around $100 \%,{ }^{18}$ it seems likely that these particles generally contain a significant amount of organic material in the core.

We have further used the values for $r_{c}, \Delta r$, and standard deviation $\sigma$ from the global fit to calculate the distribution function according to Eq. (6) and compare this result with the distribution obtained by TEM, as shown in Fig. 2. The result of this calculation is shown in Fig. 9. Two distributions are shown, the size distribution of the total radius $L\left(r_{c}+\Delta r\right)$ and the size distribution of the core $L\left(r_{c}\right)$ alone, which is simply shifted by $\Delta r=2.3 \mathrm{~nm}$, since it is not clear to what extent the shell is seen in the TEM. Although the TEM distribution is somewhat more skewed than the Schulz-Zimm distribution used in the SANS fit, the results of the two methods are in good agreement.

\section{CONCLUSIONS}

In this paper we have employed SANS on a core-shell particle with a rather thin shell $\left(r_{c} / \Delta r \approx 14\right)$. We analyzed the shell thickness and penetration of the shell by solvent molecules in terms of a contrast match experiment and found that only by a refined global fit routine reasonable parameters characterizing the system could be obtained. Thereby one fits simultaneously all data sets with a global set of parameters, which allows the separation of the influence of several strongly correlated parameters on the intensity signal. In that 
way we were able to obtain the thickness of the shell, the core radius, the distribution of the core radii, the core contrast, and the penetration of the shell by solvent (see Table II). With regard to shell properties, we found that only the product $V_{\text {shell }}\left(1-\phi_{\text {sol }}\right)$ could be determined with high accuracy, whereas the determination of the shell thickness or the penetration of solvent separately is less accurate. By chemical and spectroscopic means we could furthermore estimate the dry shell volume separately, from which in combination with the SANS results we could conclude that a significant amount of alkyl groups must be contained in the core. We further found that the core-shell particle shows a strong $q$ dependence of $\tilde{S}(q)$ at zero contrast conditions. The $\tilde{S}(q)$ increases the more we are approaching to zero contrast. This stems from a nonuniform scattering length density profile and a distribution of radii. With regard to the analytical expression for $\widetilde{S}(q)$ we found that the description by Robertus et $a l .{ }^{16}$ is most adequate and likewise the ansatz by Giacometti and co-workers ${ }^{31,32}$ based on a scaling approximation. The often used decoupling approach works reasonably well only up to volume fractions of about 0.05 at zero average contrast.

${ }^{1}$ A. Vrij and R. Tuinier, in Fundamentals of Interface and Colloid Science, edited by J. Lyklema (Elsevier, Amsterdam, 2005), Vol. IV.

${ }^{2}$ E. A. Nieuwenhuis, C. Pathmamanoharan, and A. Vrij, J. Colloid Interface Sci. 81, 196 (1981).

${ }^{3}$ R. J. Baxter, J. Chem. Phys. 49, 2770 (1968).

${ }^{4}$ J. W. Jansen, C. G. de Kruif, and A. Vrij, J. Colloid Interface Sci. 114, 471 (1986).

${ }^{5}$ J. Edwards, D. H. Everett, T. O'Sullivan, I. Pengalon, and B. Vincent, J. Chem. Soc., Faraday Trans. 1 80, 2599 (1984)

${ }^{6}$ P. W. Rouw and C. G. de Kruif, J. Chem. Phys. 88, 7799 (1988).

${ }^{7}$ C. G. de Kruif and J. A. Schouten, J. Chem. Phys. 92, 6098 (1990).

${ }^{8}$ C. G. de Kruif, W. J. Briels, R. P. May, and A. Vrij, Langmuir 4, 668 (1988)

${ }^{9}$ C. G. de Kruif, P. W. Rouw, W. J. Briels, M. H. G. Duits, A. Vrij, and R. P. May, Langmuir 5, 422 (1989).
${ }^{10} \mathrm{H}$. Verduin, Ph.D. thesis, University of Utrecht, 1996.

${ }^{11}$ M. Zackrisson, A. Stradner, P. Schurtenberger, and J. Bergenholtz, Langmuir 21, 10835 (2005).

${ }^{12}$ A. Rübe, G. Hause, K. Mäder, and J. Kohlbrecher, J. Controlled Release 107, 244 (2005).

${ }^{13}$ N. Dingenouts, S. Seelenmeyer, I. Deike, S. Rosenfeld, M. Ballauff, P. Lindner, and T. Narayanan, Phys. Chem. Chem. Phys. 3, 1169 (2001).

${ }^{14}$ J. Wagner, J. Appl. Crystallogr. 37, 750 (2004).

${ }^{15}$ T. Riley, C. R. Heald, S. Stolnik et al., Langmuir 19, 8428 (2003).

${ }^{16}$ C. Robertus, W. H. Philipse, J. G. H. Joosten, and Y. K. Levine, J. Chem. Phys. 90, 4482 (1989).

${ }^{17}$ W. Stöber, A. Fink, and E. Bohn, J. Colloid Interface Sci. 26, 62 (1968).

${ }^{18}$ A. K. van Helden, J. W. Jansen, and A. Vrij, J. Colloid Interface Sci. 81, 354 (1981).

${ }^{19}$ J. C. Thomas, J. Colloid Interface Sci. 117, 187 (1986).

${ }^{20}$ J. Kohlbrecher and W. Wagner, J. Appl. Crystallogr. 33, 804 (2000).

${ }^{21}$ U. Keiderling, Appl. Phys. A: Mater. Sci. Process. 74, S1455 (2002).

${ }^{22}$ P. Strunz, J. Saroun, U. Keiderling, A. Wiedenmann, and R. Przenioslo, J. Appl. Crystallogr. 33, 829 (2000).

${ }^{23}$ J. S. Pedersen, D. Posselt, and K. Mortensen, J. Appl. Crystallogr. 23, 321 (1990).

${ }^{24}$ H. Verduin and J. K. G. Dhont, J. Colloid Interface Sci. 172, 425 (1995).

${ }^{25}$ J. Buitenhuis, J. Kohlbrecher, M. P. Lettinga, and G. Meier (unpublished).

${ }^{26}$ M. A. Miller and D. Frenkel, Phys. Rev. Lett. 90, 135702 (2003).

${ }^{27}$ J. S. Higgins and H. C. Benoit, Polymers and Neutron Scattering (Oxford Science, Oxford, 1994)

${ }^{28}$ L. A. Feigin and D. I. Svergun, Structure Analysis by Small-Angle X-Ray and Neutron Scattering (Plenum, New York, 1987).

${ }^{29}$ J. S. Pedersen, Adv. Colloid Interface Sci. 70, 171 (1997).

${ }^{30}$ A. Vrij, J. Colloid Interface Sci. 90, 110 (1982).

${ }^{31}$ D. Gazzillo, A. Giacometti, R. G. D. Valle, E. Venuti, and F. Carsughi, J. Chem. Phys. 111, 7636 (1999).

${ }^{32}$ D. Gazzillo and A. Giacometti, Physica A 304, 202 (2002).

${ }^{33}$ A. A. Chabanov, Y. Jun, and A. J. Norris, Appl. Phys. Lett. 84, 3573 (2004).

${ }^{34}$ B. J. Maranzano and N. J. Wagner, J. Chem. Phys. 114, 10514 (2001).

${ }^{35}$ A. van Blaaderen and A. Vrij, "Synthesis and Characterisation of Colloidal Model Particles Made from Organoalkoxysilanes" in The Colloid Chemistry of Silica, Advances in Chemistry Series Vol. 234, edited by H. E. Bergna (American Chemical Society, Washington, DC, 1994).

${ }^{36}$ S. Roke, J. Buitenhuis, J. C. van Miltenburg, M. Bonn, and A. van Bladeren, J. Phys.: Condens. Matter 117, S3469 (2005). 